Pacific

Journal of

Mathematics

SHARP ISOPERIMETRIC INEQUALITIES

AND SPHERE THEOREMS

Shihshu Walter Wei And Meijun Zhu

Volume $220 \quad$ No. 1

May 2005 


\title{
SHARP ISOPERIMETRIC INEQUALITIES AND SPHERE THEOREMS
}

\author{
Shihshu WALTER Wei AND MEIJUn ZHU
}

\begin{abstract}
Various relations between sharp isoperimetric inequalities and volumes of manifolds are studied. In particular, we introduce and estimate sharp isoperimetric constants $\tau^{*}$ and $\boldsymbol{\gamma}^{*}$ corresponding to two types of isoperimetric inequalities. We show that for a complete $n$-dimensional manifold $M$ with Ricci curvature $\operatorname{Ric}(M) \geq n-1$, the volume of $M$ is close to that of $S^{n}$ if and only if $\tau^{*}$ is close to $n(n-1) /\left(2(n+2) \omega_{n}^{2 / n}\right)$ and $M$ is simply connected (for $n=2$ or 3), or $\gamma^{*}$ is close to 1 (for any $n \geq 2$ ).
\end{abstract}

\section{Introduction}

A sharp Sobolev inequality of Aubin and Li [1999] states that on an $n$-dimensional smooth, compact, connected Riemannian manifold $M$, for $p \in(1, n)$ if $n \geq 4$, or for $p \in(1, \sqrt{n}) \cup(2, n)$ if $n=2$ or 3 , and for $r>r^{*}=n p /(n+2-p)$, there exists a constant $A(p, r)>0$ depending only on $n$, the bound on the injectivity radius, and the bound on the curvature tensor and its covariant derivatives on $M$ such that, for all $\varphi \in W^{1, p}(M)$,

$$
(1-1)\left(\int_{M}|\varphi|^{p^{*}} d v\right)^{p / p^{*}} \leq K(n, p)^{p} \int_{M}|\nabla \varphi|^{p} d v+A(p, r)\left(\int_{M}|\varphi|^{r} d v\right)^{p / r},
$$

where $p^{*}=n p /(n-p)$ and

$$
K(n, p)=\frac{1}{n}\left(\frac{n(p-1)}{n-p}\right)^{(p-1) / p}\left(\frac{\Gamma(n+1)}{\Gamma\left(\frac{n}{p}\right) \Gamma\left(n+1-\frac{n}{p}\right) n \omega_{n}}\right)^{1 / n},
$$

for $\Gamma$ the gamma function, $\omega_{n}$ the volume of the unit ball in $\mathbb{R}^{n}$, and $d v$ the volume element of $M$. This inequality solves a conjecture raised by Aubin in the late 1970's; similar results were obtained independently in [Druet 1998]. It is natural

MSC2000: 58E35, 53C20, 53A99.

Keywords: isoperimetric inequality, Ricci curvature, sectional curvature, Sobolev inequality. 
to ask whether (1-1) holds for $p=1$ and $r=r^{*}$. Equivalently, does there exist, for every domain $\Omega \subset M$, a constant $C(M)$ depending on $M$ such that

$$
P^{n} \geq n^{n} \omega_{n} V^{n-1}\left(1-C(M) V^{2 / n}\right),
$$

where $P=\operatorname{vol}_{n-1} \partial \Omega$ and $V=\operatorname{vol}_{n} \Omega$ ? It is well known that (1-2) does hold for a geodesic ball with small volume; see (2-2), for example.

The case $p=1$ in (1-1) is not addressed in [Aubin and Li 1999]. On the other hand, an elegant local inequality due to Morgan and Johnson [2000] implies:

Theorem A. If the sectional curvature $K$ of $M$ is less than $K_{0}$, then an enclosure of small volume $V$ has perimeter $P$ satisfying

$$
P \geq\left(1-C K_{0} V^{2 / n}\right) P^{*},
$$

where $C$ is a constant and $P^{*}$ is the perimeter of the Euclidean ball of volume $V$.

This local result was previously only known for small geodesic balls - see (1-2) and (2-2). Equation (1-3) improved on the bound $P \geq\left(1-C^{\prime} V^{2 /(n(n+3))}\right) P^{*}$ found in [Bérard and Meyer 1982] and valid for small volume $V$.

As a consequence of (1-3) we can make the following statement, valid even when $V$ is not small, extending the Aubin-Li inequality (1-1) to the case $p=1$ and $r=r^{*}$, and initiating the study of the isoperimetric inequality (1-4):

Theorem 1.1 (An isoperimetric inequality). For every domain $\Omega \subset M$, there exists a constant $C(M)$ depending on $M$ such that

$$
P^{n} \geq n^{n} \omega_{n} V^{n-1}\left(1-C(M) V^{2 / n}\right),
$$

where $P=\operatorname{vol}_{n-1} \partial \Omega, V=\operatorname{vol}_{n} \Omega$, and $\omega_{n}$ is the volume of the unit ball in $\mathbb{R}^{n}$. (One can take, for example, $C(M)=\max \left\{n C K_{0}, \epsilon_{0}(M)^{-2 / n}\right\}$, where $C K_{0}$ is as in (1-3) and $\epsilon_{0}(M)>0$ is a constant depending on $M$ so that (1-3) holds for small $V \leq \epsilon_{0}$.)

Remark. After we completed our work, we learned that Druet [2002] had given another proof of (1-4) by a different approach.

By a standard technique involving the coarea formula and Cavalieri's principle, we see that $(1-4)$ is equivalent to the following:

Theorem 1.2 (A Sobolev inequality). There exists a constant $A=A(M)$ such that for all $\varphi \in W^{1,1}(M)$,

$$
\begin{aligned}
\left(\int_{M}|\varphi|^{n /(n-1)} d v\right)^{(n-1) / n} \leq K(n, 1) \int_{M}|\nabla \varphi| d v & \\
& +A(M)\left(\int_{M}|\varphi|^{n /(n+1)} d v\right)^{(n+1) / n},
\end{aligned}
$$

where $K(n, 1)=\lim _{p \rightarrow 1} K(n, p)=\left(n \omega_{n}\right)^{-1 / n}$. 
The isoperimetric inequality (1-4) has its roots in global analysis and partial differential equations (see, for example, [Aubin and Li 1999]). The optimal constants in (1-4), too, will have geometric and even topological applications. An immediate example is that a sharp estimate on $C(M)$ in (1-4) in two dimensions will recapture the Bernstein isoperimetric inequality [1905] on $S^{2}$,

$$
L^{2} \geq 4 \pi A\left(1-\frac{1}{4 \pi} A\right),
$$

with equality if and only if the domain in question is a disk; see Theorem 1.3(I).

Now introduce, for an $n$-dimensional, smooth, compact, connected Riemannian manifold $M$, the isoperimetric constant $\tau^{*}=\tau^{*}(M)$, defined as the constant $C(M)$ that makes (1-4) sharp:

$$
\tau^{*}:=\inf \{C(M): C(M) \text { is a constant such that }(1-4) \text { holds }\} .
$$

The constant $\tau^{*}$ depends deeply on the geometric properties of the underlying manifold $M$. In turn, it may even completely determine the metric of $M$ :

Theorem 1.3. Let $M$ be a complete, simply connected Riemannian manifold with $\operatorname{Ric}(M) \geq n-1$.

(I) The isoperimetric constant $\tau^{*}$ satisfies

$$
\tau^{*} \geq \tau_{0}:=\frac{n(n-1)}{2(n+2) \omega_{n}^{2 / n}} .
$$

For $n=2$ or 3 , we have $\tau^{*}=\tau_{0}$ if and only if $M$ is isometric to $S^{n}$ with the standard metric.

(II) For $n=2$ or 3 , if the isoperimetric constant $\tau^{*}$ is close to $\tau_{0}$, then $\operatorname{vol} M$ is close to vol $S^{n}$.

This theorem is sharp, and generalizes the Bernstein inequality (1-5). Also, the assumption of simple connectedness is necessary for the last sentence of (I), as can be seen from the example of three-dimensional real projective space, which is complete, not simply connected, and satisfies $\tau^{*}=\tau_{0}$.)

Open Problem. For $M$ of dimension $n \geq 4$, complete and simply connected, with $\operatorname{Ric}(M) \geq n-1$, does $\tau^{*}=\tau_{0}$ still imply that $M$ is isometric to the standard unit sphere $S^{n}$ ?

In Section 5 we prove that $\tau^{*}=\tau_{0}$ also for $M=S^{4}$ and $S^{5}$ :

Theorem 1.4. For any domain $\Omega$ of volume $V$ and perimeter $P$ in $S^{n}$, where $n=2,3,4$ or 5 , and with $\tau_{0}$ as in (1-7), we have

$$
P^{n} \geq n^{n} \omega_{n} V^{n-1}\left(1-\tau_{0} V^{2 / n}\right) .
$$


Open Problem. Is the isoperimetric constant still $\tau_{0}$ on the standard unit sphere $S^{n}$, for all $n \geq 6$ ? That is, does (1-8) (or equivalently (5-3) below) hold for $n \geq 6$ ?

Remark. For a complete manifold $M$ with $\operatorname{Ric}(M) \geq n-1$, the equality $\tau^{*}=\tau_{0}$ implies that $M$ is (positive) Einstein (see the proof of Theorem 1.3). This opens up the perspective of studying positive Einstein metrics via isoperimetric constants.

In high dimensions, we have an analog of Toponogov's version of S. Y. Cheng's Maximum Diameter Theorem, in the setting of the sharp isoperimetric inequality Theorem 1.3 being realized on the sphere:

Theorem 1.5. If $M$ is a complete, simply connected n-manifold of sectional curvature $\operatorname{Sec}(M) \geq 1$ and such that $\tau^{*}\left(M^{n}\right)$ is close to $\tau_{0}$, then vol $M$ is close to vol $S^{n}$ for all $n \geq 2$.

Open Problem. Does Theorem 1.5 remain true in dimensions $n \geq 4$ if one weakens the assumption that $\operatorname{Sec}(M) \geq 1$ to the assumption that $\operatorname{Ric}(M) \geq n-1$ ?

One may also investigate the converse of Theorem 1.3(II) on the estimates of $\tau^{*}$ under some assumptions on the Ricci curvature and volume of the manifold. This is related to the study of the second constant of sharp Sobolev inequalities (see, for example, [Hebey 1999]). However, we will show by an example that $\tau^{*}$ might not be close to $\tau_{0}$ even if $C \geq \operatorname{Ric}(M) \geq n-1$ and vol $M$ is close to vol $S^{n}$. Therefore, under the assumption that $M$ has bounded Ricci curvature, saying that vol $M$ is close to vol $S^{n}$ is not equivalent to saying that $\tau^{*}$ is close to $\tau_{0}$. In an attempt to solve this problem of searching for a new equivalence, we turn to the isoperimetric inequality of Gromov [1980] (see also [Chavel 1993, Theorem 6.6]):

Theorem B (Gromov's isoperimetric inequality). Given an n-dimensional compact manifold $M$ with Ric $(M) \geq n-1$ and a domain $\Omega \subset M$ with smooth boundary $\partial \Omega$, let $\Omega_{0} \subset S^{n}$ be a spherical cap such that

$$
\frac{\operatorname{vol} \Omega_{0}}{\operatorname{vol} S^{n}}=\frac{\operatorname{vol} \Omega}{\operatorname{vol} M}
$$

Then

$$
\operatorname{vol} \partial \Omega \geq \frac{\operatorname{vol} M}{\operatorname{vol} S^{n}} \cdot \operatorname{vol} \partial \Omega_{0} .
$$

Thus it makes sense to consider, for a complete manifold $M$ with $\operatorname{Ric}(M) \geq n-1$, Gromov's isoperimetric constant $\gamma^{*}=\gamma^{*}(M)$, defined by

$(1-10) \quad \gamma^{*}:=\sup \left\{\gamma(M): \operatorname{vol} \partial \Omega \geq \gamma(M) \operatorname{vol} \partial \Omega_{0}\right.$ for any domain $\left.\Omega \subset M\right\}$,

where $\partial \Omega$ is smooth and $\Omega_{0} \subset S^{n}$ is a spherical cap satisfying (1-9).

The isoperimetric constants $\tau^{*}(M)$ and $\gamma^{*}(M)$ open up a new perspective on complete manifolds $M$ with $\operatorname{Ric}(M) \geq n-1$. In particular, there are a variety of equivalent ways of stating that $\gamma^{*}$ is close to 1 , such as the following: 
Theorem 1.6. Assume that $M$ is complete with $\operatorname{Ric}(M) \geq n-1$. Then $\gamma^{*}$ is close to 1 if and only if vol $M$ is close to vol $S^{n}$ for all $n \geq 2$.

This provides a new approach to the relation vol $M \sim \operatorname{vol} S^{n}$. Other equivalent relations [Colding 1996a; 1996b; Petersen 1999] involve the Gromov-Hausdorff distance, the radius, and the $(n+1)$-st eigenvalue. As a consequence of this work, Theorem 1.5, and work of Cheeger and Colding [1997], one can conclude:

Theorem 1.7. Let $M$ be complete with $\operatorname{Ric}(M) \geq n-1$. For all $n \geq 2$, the following properties (1)-(5) are equivalent and each of them implies property (6):

(1) $\gamma^{*}$ is close to 1.

(2) vol $M$ is close to vol $S^{n}$.

(3) $M$ is Gromov-Hausdorff close to $S^{n}$.

(4) $M$ has radius close to $S^{n}$, where the radius of $M$ is that of the smallest closed metric ball that covers $M$.

(5) The $(n+1)$-st eigenvalue is close to $n$.

(6) $M$ is diffeomorphic to $S^{n}$.

Corollary 1.8. Let $M$ be complete and simply connected with $\operatorname{Sec}(M) \geq 1$ if $n \geq 2$, or $\operatorname{Ric}(M) \geq n-1$ if $n=2$ or 3 . Then the properties (2)-(6) below are equivalent, each of them is implied by property (1), and each implies properties (7)-(9):

(1) $\tau^{*}$ is close to the constant $\tau_{0}:=\frac{n(n-1)}{2(n+2) \omega_{n}^{2 / n}}$.

(2) $\gamma^{*}$ is close to 1 .

(3) vol $M$ is close to vol $S^{n}$.

(4) $M$ is Gromov-Hausdorff close to $S^{n}$.

(5) $M$ has radius close to $S^{n}$.

(6) The $(n+1)$-st eigenvalue is close to $n$.

(7) $M$ is diffeomorphic to $S^{n}$.

(8) $M$ has diameter close to $S^{n}$.

(9) The first eigenvalue is close to $n$.

\section{Proof of Theorem 1.3}

We begin with the asymptotic formulas for the perimeter $P$ and volume $V$ of a geodesic ball $B_{r}(\bar{x})$ of scalar curvature $\operatorname{Scal}_{\bar{x}}(M)$ about a point $\bar{x}$ (see, for example, [Gallot et al. 1987, Theorem 3.98]):

$$
\frac{P^{n}}{V^{n-1}}=n^{n} \omega_{n}\left(1-\frac{\operatorname{Scal}_{\bar{x}}(M)}{2(n+2)} r^{2}+O\left(r^{4}\right)\right) .
$$


Thus, for a domain that is a geodesic ball $B_{r}(\bar{x})$ with small volume,

$$
P^{n}=n^{n} \omega_{n} V^{n-1}\left(1-\frac{\operatorname{Scal}_{\bar{x}}(M)}{2(n+2) \omega_{n}^{2 / n}} V^{2 / n}+o(1) V^{3 / n}\right),
$$

where $o(1)$ is small and tends to 0 as $V \rightarrow 0$.

Since $\operatorname{Ric}(M) \geq n-1$, we have $\operatorname{Scal}_{\bar{x}}(M) \geq n(n-1)$ at any point of $M$; thus by (1-4) and (1-6),

$$
\tau^{*} \geq \frac{\operatorname{Scal}_{\bar{x}}(M)}{2(n+2) \omega_{n}^{2 / n}} \geq \frac{n(n-1)}{2(n+2) \omega_{n}^{2 / n}}=\tau_{0} .
$$

For $n=2$ or 3, if $\tau^{*}=\tau_{0}$ we know from (2-3) that $\operatorname{Scal}_{x}(M) \leq n(n-1)$ at any point $x$ in $M$; thus $\operatorname{Ric}(M)=n-1$. This in turn implies that $M$ has constant sectional curvature $K=1$, and is therefore isometric to the standard unit sphere. On the other hand, for $S^{2}$, due to Gromov's isoperimetric inequality (Theorem B), we need only prove that for any spherical cap domain $\Omega$, the equality in (1-7) holds. This is obvious, since in terms of the spherical coordinate $\theta$ (which measures down from the north pole) we have $L=2 \pi \sin \theta$ and $A=2 \pi \int_{0}^{\theta} \sin \alpha d \alpha$ for $0 \leq \theta \leq \pi$. It follows that $\tau^{*}=1 / 4 \pi$, and we recapture the standard Bernstein isoperimetric inequality (1-5). In the case of $S^{3}$, due to Theorem B, it suffices to prove that, for any spherical cap domain $\Omega$,

$$
P^{3} \geq 36 \pi V^{2}\left(1-\frac{3}{5}\left(\frac{4 \pi}{3}\right)^{-2 / 3} V^{2 / 3}\right) .
$$

In terms of the spherical coordinate function, for $0 \leq \theta \leq \pi$,

$$
P=4 \pi \sin ^{2} \theta \quad \text { and } \quad V=4 \pi \int_{0}^{\theta} \sin ^{2} \alpha d \alpha .
$$

Viewing $P$ as a function of $V$, we define

$$
f(V)=P^{3}-36 \pi V^{2}\left(1-\frac{3}{5}\left(\frac{4 \pi}{3}\right)^{-2 / 3} V^{2 / 3}\right) .
$$

Direct computation yields

$$
\frac{d P}{d V}=\frac{2 \cos \theta}{\sin \theta}, \quad \frac{d^{2} P}{d V^{2}}=\frac{-8 \pi}{\left(4 \pi \sin ^{2} \theta\right)^{2}}=\frac{-8 \pi}{P^{2}},
$$

so that

$$
\frac{d f(V)}{d V}=24 \pi^{2}\left(4 \sin ^{3} \theta \cos \theta-3(2 \theta-\sin 2 \theta)+\frac{12}{5}\left(\frac{4}{3}\right)^{-2 / 3}(2 \theta-\sin 2 \theta)^{5 / 3}\right)
$$

and

$$
\frac{d^{2} f(V)}{d V^{2}}=-96 \pi\left(\sin ^{2} \theta-\left(\frac{4}{3}\right)^{-2 / 3}(2 \theta-\sin 2 \theta)^{2 / 3}\right)
$$


Specializing for $\theta=0$ (so $V=0$ and $P=0$ ) we have

$$
f(0)=\frac{d f}{d V}(0)=\frac{d^{2} f}{d V^{2}}(0)=0,
$$

Note that $d^{2} f(V) / d V^{2}$ has the same sign as

$$
\mu(\theta)=2 \theta-\sin 2 \theta-\frac{4}{3} \sin ^{3} \theta .
$$

One easily checks that $\mu(0)=0$, and $\mu^{\prime}(\theta)=4 \sin ^{2} \theta-4 \sin ^{2} \theta \cos \theta>0$ for $\theta \in(0, \pi)$. It follows that $f(V) \geq 0$. This completes the proof of part (I).

To prove part (II), first observe that vol $M \leq$ vol $S^{n}$ by the Bishop volume comparison theorem [Bishop and Crittenden 1964]. For $n=2$, if the statement were not true, there would exist $\delta>0$ such that vol $M^{2} \leq 4 \pi-\delta$ for some manifold $M^{2}$ with

$$
\tau^{*}\left(M^{2}\right)-\frac{1}{4 \pi} \leq \frac{\delta}{8 \pi(4 \pi-\delta)} .
$$

We then choose $\Omega=M \backslash B_{\epsilon}$, where $B_{\epsilon}$ is a small geodesic ball of radius $\epsilon$ in $M$. For such a domain $\Omega$,

$$
P^{2} \geq 4 \pi V\left(1-\left(\frac{1}{4 \pi}+\frac{\delta}{8 \pi(4 \pi-\delta)}\right) V\right)
$$

which would imply that $0 \geq \frac{1}{2} \delta V>0$ as $\epsilon \rightarrow 0$, a contradiction.

For $n=3$, we need the following lemma, which is a slight variation on a convergence theorem due to Petersen [1998, 10.5.4, Theorem 5.10]:

Lemma 2.1. Given $n \geq 2$ and $\lambda>0$, there is an $\epsilon=\epsilon(n, \lambda)>0$ such that any closed, simply connected Riemannian n-manifold $(M, g)$ with $|\operatorname{Sec}(M)-\lambda| \leq \epsilon$ is $C^{1, \alpha}$-close to a metric of constant curvature $\lambda$.

Proof of Lemma 2.1. By the Bonnet Theorem [1855], $\operatorname{Sec}(M) \geq \lambda-\epsilon$ implies $\operatorname{diam}(M) \leq \pi / \sqrt{\lambda-\epsilon}$. Then, for $n \geq 3$, replacing Cheeger's lemma (see [Petersen 1998, pages 300-301]) by Klingenberg's Theorem [1959], which implies that the injectivity radius is at least $\pi / \sqrt{\lambda+\varepsilon}$, one may readily modify [Petersen 1998, proof on page 312] to deduce the conclusion. For $n=2$ instead of Klingenberg's Theorem one can use Synge's Theorem and [Carmo 1992, Proposition 3.4, p. 281].

From $(2-3)$ we can see that $\operatorname{Ric}(M) \rightarrow 2$ as $\tau^{*} \rightarrow \frac{3}{5}\left(\frac{3}{4 \pi}\right)^{2 / 3}$. This implies that $\operatorname{Sec}(M) \rightarrow 1$, since on a 3-manifold $M$ and for some constant $K_{0}$, there is equivalence between $\operatorname{Ric}(M) \equiv 2 K_{0}$ and $\operatorname{Sec}(M) \equiv K_{0}$. Then from Lemma 2.1 we know that the metric of $M$ converges to the standard metric of $S^{3}$ in the $C^{1, \alpha}$ topology as $\tau^{*} \rightarrow \frac{3}{5}\left(\frac{3}{4 \pi}\right)^{2 / 3}$. This implies that vol $M \rightarrow \operatorname{vol} S^{3}=2 \pi^{2}$, completing the proof of part (II) and of Theorem 1.3. 
Remark. Conceivably, estimates on $\tau^{*}$ may yield estimates on the first eigenvalue. For instance, assuming that $M$ is complete and simply connected, and that $\operatorname{Ric}(M) \geq n-1$, then $\lambda_{1}$ is close to $n$ if $\tau^{*}$ is close to $\tau_{0}$ for $n=2$ or 3 . This can be proved as follows. According to Theorem 1.3 we know that vol $M$ is close to vol $S^{n}$, thus $\operatorname{rad}(M)$ is close to $\pi$ (see, for example, [Petersen 1999]). This of course yields that diam $(M)$ is close to $\pi$. Then due to a theorem of Cheng [1975] we know that $\lambda_{1}$ is close to $n$ (see Corollary 1.8).

\section{A small manifold with large isoperimetric constant}

We show that the converse of Theorem 1.3(II) is not true. Assume that $\operatorname{Ric}(M) \geq$ $n-1$. For a geodesic ball $B_{r}(\bar{x})$ with small volume, we recall (2-2)

$$
P^{n}=n^{n} \omega_{n} V^{n-1}\left(1-\frac{\operatorname{Scal}_{\bar{x}}(M)}{2(n+2) \omega_{n}^{2 / n}} V^{2 / n}+o(1) V^{3 / n}\right),
$$

where $\operatorname{Scal}_{\bar{x}}(M)$ is the scalar curvature at point $\bar{x}$ and $o(1) \rightarrow 0$ as $V \rightarrow 0$.

One can check that, for $n=2$,

$$
\frac{n(n-1)}{2(n+2) \omega_{n}^{2 / n}}=\left((n+1) \omega_{n+1}\right)^{-2 / n} .
$$

If vol $M \rightarrow \operatorname{vol} S^{2}$ implied that $\tau^{*} \rightarrow 1 / 4 \pi$, then $\operatorname{Scal}_{\bar{x}}(M)$ would be less than $2+\delta$ as vol $M \rightarrow \operatorname{vol} S^{2}$, for any $\delta>0$. But the following example shows that this is impossible.

Example 3.1. For any small positive $\epsilon$ (less than $\frac{1}{100}$, say), define a $C^{2}$-smooth function by

$$
f_{\epsilon}(x)=\left\{\begin{array}{llr}
\sqrt{1-(x-\epsilon)^{2}} & \text { if } & -1+\epsilon \leq x \leq-\epsilon, \\
h_{\epsilon}(x) & \text { if } & -\epsilon \leq x \leq \epsilon, \\
\sqrt{1-(x+\epsilon)^{2}} & \text { if } & \epsilon \leq x \leq 1-\epsilon
\end{array}\right.
$$

where $h_{\epsilon}$ is a symmetric function to be determined. Direct computation shows that

$$
\begin{aligned}
f_{\epsilon}^{\prime}(-\epsilon) & =-f_{\epsilon}^{\prime}(\epsilon)=2 \epsilon+o_{\epsilon}(1) \epsilon^{2}, \\
f_{\epsilon}^{\prime \prime}(\epsilon) & =f_{\epsilon}^{\prime \prime}(-\epsilon)=-1+o_{\epsilon}(1) \epsilon,
\end{aligned}
$$

where $o_{\epsilon}(1)$ is small and tends to 0 as $\epsilon \rightarrow 0$. For a small $\epsilon>0$, we choose a negative continuous symmetric function $g_{\epsilon}$ satisfying $g_{\epsilon}( \pm \epsilon)=f_{\epsilon}^{\prime \prime}(\epsilon)=f_{\epsilon}^{\prime \prime}(-\epsilon)$, $g_{\epsilon}^{\prime}(x)<0$ for $-\epsilon \leq x<0$,

$$
-5 \leq \min _{-\epsilon \leq x \leq \epsilon} g_{\epsilon}(x) \leq-2 \text { and } \int_{-\epsilon}^{\epsilon} g_{\epsilon} d x=2 f_{\epsilon}^{\prime}(\epsilon) .
$$


The existence of such a function is guaranteed by (3-1). We then define $h_{\epsilon}$ to be a symmetric function such that $h_{\epsilon}(-\epsilon)=\sqrt{1-4 \epsilon^{2}}$ and

$$
h_{\epsilon}^{\prime}(x)=\int_{-\epsilon}^{x} g_{\epsilon}(s) d s+f_{\epsilon}^{\prime}(-\epsilon) \text { for }-\epsilon<x \leq 0 .
$$

Let $M_{\epsilon}$ be the surface obtaining by rotating $y=f_{\epsilon}(x)$ around the $x$-axis. Recall that the Gaussian curvature $K_{\epsilon}$ is given by

$$
K_{\epsilon}=-\frac{f_{\epsilon}^{\prime \prime}}{f_{\epsilon}\left(1+\left(f_{\epsilon}^{\prime}\right)^{2}\right)^{2}},
$$

where differentiation is with respect to $x$. It is easy to check that $K_{\epsilon} \geq 1+o_{\epsilon}(1)$ and vol $M_{\epsilon}=\operatorname{vol} S^{2}+o_{\epsilon}(1)$, but $K_{\epsilon}$ is greater than $\frac{3}{2}+o_{\epsilon}(1)$ at the equator of $M_{\epsilon}$, so the scalar curvature $\operatorname{Scal}_{x}\left(M_{\epsilon}\right)$ is at least $3+o_{\epsilon}(1)$ at the equator of $M_{\epsilon}$. By rescaling, one easily obtains a sequence of manifolds $M_{\epsilon}$ with Gaussian curvatures $K_{\epsilon} \geq 1$ and volumes vol $M_{\epsilon} \rightarrow \operatorname{vol} S^{2}$, but with scalar curvatures $\operatorname{Scal}_{x}\left(M_{\epsilon}\right)>\frac{5}{2}$ at some points.

\section{Proof of proximity results}

Proof of Theorem 1.5. In view of (2-3), $\operatorname{Sec}(M) \rightarrow 1$ as $\tau^{*} \rightarrow \tau_{0}$. It then follows from Lemma 2.1 that the metric of $M$ converges to the standard metric of $S^{n}$ in the $C^{1, \alpha}$ topology as $\tau^{*} \rightarrow \tau_{0}$. This implies that vol $M \rightarrow \operatorname{vol} S^{n}$.

Proof of Theorem 1.6. Let $M$ be complete with $\operatorname{Ric}(M) \geq n-1$. We claim that

$$
\gamma^{*} \leq 1
$$

If not, there is $\delta>0$ such that

$$
\operatorname{vol} \partial \Omega \geq(1+\delta) \operatorname{vol} \partial \Omega_{0}
$$

for any smooth domain $\Omega \subset M$. Now, [Morgan and Johnson 2000, Theorem 3.4] says that given $V$, the manifold $M$ has regions of volume $V$ and perimeter at most equal to the perimeter $P_{0}(V)$ of a ball of volume $V$ in $S^{n}$. Choose $V=\frac{1}{2} \operatorname{vol} M$; since vol $M \leq \operatorname{vol} S^{n}$, we know that

$$
P(V) \leq P_{0}(V) \leq \operatorname{vol} S^{n-1} .
$$

However, from (4-2) we have

$$
P(V) \geq(1+\delta) \operatorname{vol} S^{n-1},
$$

which is a contradiction. This proves (4-1).

If vol $M$ is close to vol $S^{n}$, we know from Theorem B that

$$
\gamma^{*} \geq \frac{\operatorname{vol} M}{\operatorname{vol} S^{n}} \rightarrow 1 \text {. }
$$


Combining this with (4-1) we get $\gamma^{*} \rightarrow 1$.

Conversely, if $\gamma^{*} \rightarrow 1$, we claim vol $M \rightarrow \operatorname{vol} S^{n}$. Otherwise, there is $\delta>0$ such that vol $M \leq \operatorname{vol} S^{n}-\delta$. Choose $V=\frac{1}{2} \operatorname{vol} M$ in [Morgan and Johnson 2000, Theorem 3.4] and let $R$ be the region whose perimeter is $P(V)$; then

$$
P(V) \leq P_{0}(V) \leq(1-\epsilon) \text { vol } S^{n-1}
$$

for some fixed $\epsilon=\epsilon(\delta)>0$, since vol $M \leq \operatorname{vol} S^{n}-\delta$. Thus

$$
\operatorname{vol} \partial R \leq(1-\epsilon) \operatorname{vol} \partial R_{0},
$$

which contradicts the fact that $\gamma^{*} \rightarrow 1$.

\section{Spheres in dimensions up to 5: Proof of Theorem 1.4}

Thanks to Gromov's isoperimetric inequality, to prove Theorem 1.4 we need only show that (1-8) holds for any spherical cap domain $\Omega$ in $S^{n}$ for $n=4$ or 5 (the cases $n=2,3$ being covered by Theorem 1.3.

For $n=4$, we must prove $P^{4} \geq 4^{4} \omega_{4} V^{3}\left(1-\omega_{4}^{-1 / 2} V^{1 / 2}\right)$, where $\omega_{4}=\pi^{2} / 2$. Using the spherical coordinate $\theta$ that measures angles down from the north pole, we know that, for $0 \leq \theta \leq \pi$,

$$
P=2 \pi^{2} \sin ^{3} \theta \quad \text { and } \quad V=2 \pi^{2} \int_{0}^{\theta} \sin ^{3} \alpha d \alpha .
$$

Viewing $P$ as a function of $V$, we define

$$
f(V)=P^{4}-4^{4} \omega_{4} V^{3}\left(1-\left(\omega_{4}\right)^{-1 / 2} V^{1 / 2}\right) .
$$

Direct computation yields

$$
\frac{d f(V)}{d V}=32 \pi^{6}\left(3 \sin ^{8} \theta \cos \theta-48 A^{2}+112 A^{5 / 2}\right),
$$

where $A=A(\theta)=\int_{0}^{\theta} \sin ^{3} \alpha d \alpha$. Since $f(0)=0$ and $d V / d \theta \geq 0$, it suffices to show that $f_{1}(\theta):=d f(V) / d V \geq 0$ for any $\theta \in(0, \pi)$.

Again, since $f_{1}(0)=0$, it is enough to show that $d f_{1}(\theta) / d \theta \geq 0$ for any $\theta \in$ $(0, \pi)$. Equivalently, it suffices to show, for any $\theta \in(0, \pi)$, that

$$
f_{2}(\theta):=24 \sin ^{4} \theta \cos ^{2} \theta-3 \sin ^{6} \theta-96 A+280 A^{3 / 2} \geq 0 .
$$

Note again that since $f_{2}(0)=0$, it is enough to show that $d f_{2}(\theta) / d \theta \geq 0$ for any $\theta \in(0, \pi)$. Equivalently, we only need to show

$$
f_{3}(\theta):=162 \cos ^{3} \theta-66 \cos \theta-96+420 A^{1 / 2} \geq 0
$$


for $\theta \in(0, \pi)$. Since $A$ is an increasing function of $\theta$, we can check, for $\theta \geq \pi / 2$, that

$$
f_{3}(\theta) \geq 420 A^{1 / 2} \pi / 2-258 \geq 0 .
$$

To prove (5-1) for $\theta \leq \pi / 2$, it is sufficient to show that

$$
g_{1}(\theta)=420^{2} A-\left(162 \cos ^{3} \theta-66 \cos \theta-96\right)^{2} \geq 0,
$$

for $\theta \in(0, \pi / 2)$. Again, since $g_{1}(0)=0$, it is enough to prove that $d g_{1} / d \theta \geq 0$ for $\theta \in(0, \pi / 2)$. Equivalently, we need only show, for $\theta \in(0, \pi / 2)$, that

$(5-2) g_{2}(\theta):=420^{2} \sin ^{2} \theta-2\left(162 \cos ^{3} \theta-66 \cos \theta-96\right)\left(66-486 \cos ^{2} \theta\right) \geq 0$.

To check this, we have, for $\theta \in(0, \pi / 2)$, and setting $s:=\sin \theta, c:=\cos \theta$,

$$
\begin{aligned}
420^{2} s^{2}-2(162 & \left.c^{3}-66 c-96\right)\left(66-486 c^{2}\right) \\
& =420^{2} s^{2}-2\left(-162 c s^{2}+96(c-1)\right)\left(66-486 c^{2}\right) \\
& =420^{2} s^{2}+324 \cdot 66 c s^{2}-324 \cdot 486 c^{3} s^{2}-192(1-c)\left(486 c^{2}-66\right) \\
& \geq 420^{2} s^{2}+324 \cdot 66 c s^{2}-324 \cdot 486 c s^{2}-192(1-c)\left(486 c^{2}-66\right) \\
& =420^{2} s^{2}-324 \cdot 420 c s^{2}-192(1-c)\left(486 c^{2}-66\right) \\
& \geq 420^{2} s^{2}-324 \cdot 420 s^{2}-192(1-c)\left(486 c^{2}-66 c^{2}\right) \\
& =420 \cdot 96 \cdot\left(s^{2}-2(1-c) c\right) \\
& =420 \cdot 96 \cdot 4 \sin ^{2}(\theta / 2) \cdot\left(\cos ^{2}(\theta / 2)-c^{2}\right) \geq 0,
\end{aligned}
$$

proving the case $n=4$.

For general $n$, we note that $(1-8)$ is equivalent to the integral inequality

$$
\sin ^{n(n-1)} \theta \geq n^{n-1} A^{n-1}-\frac{n^{n+(2 / n)}(n-1)}{2(n+2)} A^{n-1+(2 / n)}
$$

for $\theta \in[0, \pi]$, where

$$
A=\int_{0}^{\theta} \sin ^{n-1} \alpha d \alpha .
$$

For $n=5$, we can follow the same argument used for $n=4$ and find that it is enough to show that

$$
f_{4}(\theta)=128 \sin ^{4} \theta-187 \sin ^{2} \theta+5^{2 / 5} \cdot 11 \cdot 17 \cdot A^{2 / 5} \geq 0
$$

for $\theta \in[0, \pi]$. Notice that $128 \sin ^{4} \theta-187 \sin ^{2} \theta \leq 0$, and so it suffices to prove

$$
g(\theta)=5^{2} \cdot 11^{5} \cdot 17^{5} \cdot A^{2}+\left(128 \sin ^{4} \theta-187 \sin ^{2} \theta\right)^{5} \geq 0 \quad \text { for } \theta \in[0, \pi] .
$$

Since $g(0)=0$, it is enough to show that, for $\theta \in[0, \pi]$, and with $s, c$ as before, $g^{\prime}(\theta)=2 \cdot 5^{2} \cdot 11^{5} \cdot 17^{5} \cdot A \cdot s^{4}+5\left(128 s^{4}-187 s^{2}\right)^{4} \cdot\left(4 \cdot 128 s^{3} c-2 \cdot 187 s c\right) \geq 0$. 
Let $g_{1}(\theta)=5 \cdot 11^{5} \cdot 17^{5} \cdot A+\left(128 s^{3}-187 s\right)^{4} \cdot\left(2 \cdot 128 s^{3} c-187 s c\right)$. Note that $g_{1}(\theta)$ has the same sign as $g^{\prime}(\theta)$ and that $g_{1}(0)=0$, so we need only show that $g_{1}^{\prime}(\theta) \geq 0$. Let

$$
\begin{aligned}
g_{2}(\theta)=5 \cdot 11^{5} \cdot 17^{5} & +4 \cdot\left(128 s^{2}-187\right)^{3}\left(3 \cdot 128 s^{2} c-187 c\right)\left(2 \cdot 128 s^{2} c-187 c\right) \\
& +\left(128 s^{2}-187\right)^{4}\left(6 \cdot 128 s^{2} c^{2}-2 \cdot 128 s^{4}-187 c^{2}+187 s^{2}\right) .
\end{aligned}
$$

Note that $g_{2}(\theta)$ has the same sign as $g_{1}^{\prime}(\theta)$ for $\theta \in[0, \pi]$. Then, by means of some delicate computations, we can check that $g_{2}(\theta) \geq 0$ for $\theta \in[0, \pi]$. It should be pointed out that $g_{2}^{\prime}(\theta)$ is no longer nonnegative for $\theta \in[0, \pi]$.

\section{Acknowledgment}

The authors thank the referee for valuable comments and remarks, and Silvio Levy and Nicholas Jackson for a superb job in editing the article.

\section{References}

[Aubin and Li 1999] T. Aubin and Y. Y. Li, "On the best Sobolev inequality", J. Math. Pures Appl. (9) 78:4 (1999), 353-387. MR 2000e:46041 Zbl 0944.46027

[Bérard and Meyer 1982] P. Bérard and D. Meyer, "Inégalités isopérimétriques et applications", Ann. Sci. École Norm. Sup. (4) 15:3 (1982), 513-541. MR 84h:58147 Zbl 0489.35058

[Bernstein 1905] F. Bernstein, "Über die isoperimetrische Eigenschaft des Kreises auf der Kugeloberfläche und in der Ebene", Math. Ann. 60 (1905), 117-136.

[Bishop and Crittenden 1964] R. L. Bishop and R. J. Crittenden, Geometry of manifolds, Pure and Applied Mathematics 15, Academic Press, New York, 1964. MR 29 \#6401 Zbl 0132.16003

[Bonnet 1855] O. Bonnet, "Sur quelques propriétés des lignes géodésiques", C. R. Acad. Sci. Paris 40 (1855), 1311-1313.

[Carmo 1992] M. P. do Carmo, Riemannian geometry, Birkhäuser, Boston, 1992. MR 92i:53001 Zbl 0752.53001

[Chavel 1993] I. Chavel, Riemannian geometry — a modern introduction, Cambridge Tracts in Mathematics 108, Cambridge University Press, Cambridge, 1993. MR 95j:53001 Zbl 0810.53001

[Cheeger and Colding 1997] J. Cheeger and T. H. Colding, "On the structure of spaces with Ricci curvature bounded below, I”, J. Diff. Geom. 46:3 (1997), 406-480. MR 98k:53044 Zbl 0902.53034

[Cheng 1975] S. Y. Cheng, "Eigenvalue comparison theorems and its geometric applications", Math. Z. 143:3 (1975), 289-297. MR 51 \#14170 Zbl 0329.53035

[Colding 1996a] T. H. Colding, "Shape of manifolds with positive Ricci curvature", Invent. Math. 124:1-3 (1996), 175-191. MR 96k:53067 Zbl 0871.53027

[Colding 1996b] T. H. Colding, "Large manifolds with positive Ricci curvature", Invent. Math. 124:1-3 (1996), 193-214. MR 96k:53068 Zbl 0871.53028

[Druet 1998] O. Druet, "Optimal Sobolev inequalities of arbitrary order on compact Riemannian manifolds”, J. Funct. Anal. 159:1 (1998), 217-242. MR 99m:53076 Zbl 0923.46035

[Druet 2002] O. Druet, "Isoperimetric inequalities on compact manifolds", Geom. Dedicata 90 (2002), 217-236. MR 2003a:53045 Zbl 1025.58014 
[Gallot et al. 1987] S. Gallot, D. Hulin, and J. Lafontaine, Riemannian geometry, Universitext, Springer, Berlin, 1987. MR 88k:53001 Zbl 0636.53001

[Gromov 1980] M. Gromov, "Paul Lévy's isoperimetric inequality", preprint M/80/320, Inst. Haut Étud. Sci., 1980.

[Hebey 1999] E. Hebey, Nonlinear analysis on manifolds: Sobolev spaces and inequalities, Courant Lecture Notes in Mathematics 5, New York University Courant Institute of Mathematical Sciences, New York, 1999. MR 2000e:58011 Zbl 0981.58006

[Klingenberg 1959] W. Klingenberg, "Contributions to Riemannian geometry in the large", Ann. of Math. (2) 69 (1959), 654-666. MR 21 \#4445 Zbl 0133.15003

[Morgan and Johnson 2000] F. Morgan and D. L. Johnson, "Some sharp isoperimetric theorems for Riemannian manifolds”, Indiana Univ. Math. J. 49:3 (2000), 1017-1041. MR 2002e:53043 Zbl 1021.53020

[Petersen 1998] P. Petersen, Riemannian geometry, Graduate Texts in Mathematics 171, SpringerVerlag, New York, 1998. MR 98m:53001 Zbl 0914.53001

[Petersen 1999] P. Petersen, "On eigenvalue pinching in positive Ricci curvature", Invent. Math. 138:1 (1999), 1-21. MR 2001e:53032 Zbl 0988.53011

Received February 5, 2004. Revised August 3, 2004.

\author{
SHIHSHU WALTER WeI \\ DEPARTMENT OF MATHEMATICS \\ UNIVERSITY OF OKLAHOMA \\ NORMAN, OK 73019 \\ UNITED STATES \\ wwei@math.ou.edu \\ Meijun Zhu \\ DEPARTMENT OF MATHEMATICS \\ UNIVERSITY OF OKLAHOMA \\ NORMAN, OK 73019 \\ UNITED STATES \\ mzhu@math.ou.edu
}

